Proceedings of Magneto-Optical Recording International Symposium '92, J. Magn. Soc. Jpn., Vol. 17, Supplement No. S1 (1993), pp. 17-22 (1) 1993 by The Magnetics Society of Japan

\title{
STUDY OF Co-BASED MULTILAYERS BY BRILLOUIN LIGHT SCATTERING
}

\author{
B. Hillebrands, J.V. Harzer, G. Güntherodt \\ 2. Physikalisches Institut, RWTH Aachen, Templergraben 55, 5100 Aachen, Germany \\ J.R. Dutcher \\ Department of Physics, University of Guelph, Guelph, Ontario, N1G 2W1, Canada
}

\begin{abstract}
We present two major aspects of magnetic anisotropies in Co-based multilayers. Within the framework of a continuum free energy calculation we show that the experimentally observed large uniaxial in-plane anisotropy in epitaxial single-crystal (110)-oriented $\mathrm{Co} / \mathrm{Pt}$ and $\mathrm{Co} / \mathrm{Pd}$ superlattices is caused by magnetoelastic interaction. From Brillouin light scattering experiments the lateral and layer-to-layer variations of anisotropies in $\mathrm{Co} / \mathrm{Pd}$ - and $\mathrm{Co} / \mathrm{Au}-$ multilayers are analyzed on the length scale of spin-wave excitations $(\approx 3000 \AA)$, which is also a characteristic length scale for domain formation in magneto-optic recording.
\end{abstract}

KEYWORDS: magnetic anisotropies, multilayers, magnetoelastic interaction, roughness, Brillouin light scattering

\section{INTRODUCTION}

During the last decade magnetic multilayered structures have been studied extensively and their applicability for magneto-optic storage media and magnetic field sensors has successfully been demonstrated [1-9]. Although technological progress has been made in advancing the magneto-optic storage characteristics of multilayered systems like $\mathrm{Co} / \mathrm{Pt}$ and $\mathrm{Co} / \mathrm{Pd}$ [6-9], yielding media already suitable for device production, the underlying physical mechanisms, in particular the origin of the observed large perpendicular anisotropy still remain largely undiscovered. There is still much effort needed in order to correlate the magnetic properties, in particular magnetic anisotropies, with detailed electronic and structural information.

This paper will focus mainly on two basic aspects of magnetic anisotropy: i) In the model system of epitaxially grown $\mathrm{Co} / \mathrm{Pd}$ and $\mathrm{Co} / \mathrm{Pt}$ multilayer structures with (110)-layer orientation the observed large in-plane anisotropy can unambiguously be identified as originating from magnetoelastic interaction [10]. ii) Spatial variations in anisotropy fields, as they are of importance for domain wall motion and stability, are characterized on the length scale of $3000 \AA$ by observing the propagation properties of spin waves [11]. This length scale is also a characteristic length scale for domain sizes in magneto-optic recording.

For the determination of magnetic quantities we use the Brillouin light scattering technique, which is inelastic scattering of light from propagating spin wave excitations [12-15]. The spin wave frequencies depend on the strength and the direction of the externally applied field, the strength and direction of the saturation magnetization, the strength and direction of the spin-wave wavevector as determined by the scattering geometry, on magnetic torques caused by anisotropies as well as on the strength of intra- and interlayer exchange interactions [15]. The magnetic properties are obtained by a fit of suitable models to the experimentally obtained spin wave dispersion properties with the magnetic quantities (saturation magnetization, anisotropy constants, exchange constants, etc.) as fit parameters [12,15].

The Brillouin light scattering experiments were performed using a computer-controlled Sandercock-type $(3+3)$-pass tandem Fabry-Perot interferometer in backscattering geometry [16]. $p$-polarized light of up to $150 \mathrm{~mW}$ from a single-mode $514.5 \mathrm{~nm} \mathrm{Ar}{ }^{+}$ion laser source is focused onto the sample with an angle of incidence of $45^{\circ}$. The laser spot diameter (probing area) is $\approx 100 \mu \mathrm{m}$. The light inelastically scattered from spin wave excitations is analyzed in the depolarized configuration in order to suppress phonon signals. A magnetic field of up to $20 \mathrm{kG}$ is applied parallel to the layer planes and perpendicular to the wave vector of the spin waves tested in the experiment.

\section{LARGE IN-PLANE ANISOTROPIES IN (110)-ORIENTED MULTILAYERS}

For (110)-oriented epitaxial $\mathrm{Co} / \mathrm{Pt}$ - and $\mathrm{Co} / \mathrm{Pd}$-multilayer structures a large in-plane uniaxial anisotropy contribution with the easy axis along the in-plane [001]-direction has been found [10]. This is demonstrated in Fig. 1.
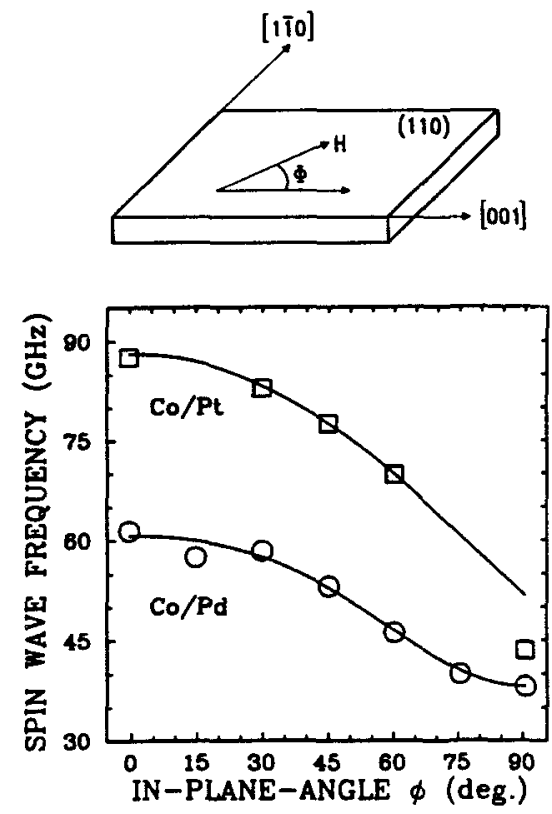

Fig. 1: Spin wave frequencies of (110)-oriented $\mathrm{Co} / \mathrm{Pt}$ and $\mathrm{Co} / \mathrm{Pd}$ multilayers vs. the in-plane direction of an applied field of $12 \mathrm{kG}$. Upper part: geometry. Lower part: spin wave frequencies of a $9.9 \AA \mathrm{Co} / 27.7 \AA \mathrm{Pt}$ - (squares) and a $12.3 \AA \mathrm{Co} / 10 \AA \mathrm{Pd}$-multilayer (circles) structure as a function of the in-plane angle $\phi$ of the direction of the applied field with the in-plane [001]-axis. The full lines are a fit to the data with parameters as listed in Table 1.

In the lower part the measured spin wave frequencies are plotted as a function of the in-plane angle $\phi$ of the direction of the applied field with the [001]-direction. The geometry is shown in the upper part of Fig. 1. The spin 
wave modes show a frequency variation of about a factor of two for the Co/Pt and of 1.5 for the Co/Pd multilayer sample. The highest frequencies correspond to the magnetically easy direction of the magnetization, which for both systems is along the $[001]$-axis in plane. From this finding a magnetocrystalline origin for the observed anisotropy can unambiguously be excluded, since for fcc-Co the easy axis would be aligned along the [111]-axis. There is no indication in the data of Fig. 1 for such a contribution.

We will now show that this contribution is of magnetoelastic origin. For the calculation we will idealize the system by assuming that the superlattice structure is coherently strained by the lattice mismatch at the interfaces. The elastic strain fields are calculated by minimizing the elastic free energy of the system and by solving for the elastic boundary conditions at the interfaces [10]. We note that this calculation yields limiting values for the strains, which however might be partly released in a real system due to dislocation formation.

As a general result we obtain that in a superlattice structure the elastically softer material accomodates the larger fraction of interface strains. In particular in $\mathrm{Co} / \mathrm{Pt}$ superlattices the $C_{0}$ layers contain the larger part of strains which in turn (see below) increases the corresponding magnetic anisotropy contributions.

The anisotropy contributions are obtained by a comparison of the magnetoelastic free energy

$$
F_{m e}=\sum_{i, j} b_{i j} \alpha_{i}^{2} \epsilon_{j}
$$

to a general anisotropy energy expression of the form:

$$
F_{\text {aniso }}=-\mathrm{K}_{\mathrm{s}} \alpha_{3}^{2}-\mathrm{K}_{\mathrm{p}} \alpha_{1}^{2}
$$

Here $\left(b_{i j}\right)$ is the magnetoelastic tensor, $\alpha_{i}$ are the direction cosines of the magnetization, and $\epsilon_{j}$ are the strains within the Co layers. We assume that the coordinate system is oriented with the $\hat{x}_{3}$-axis along the stack axis and the $\hat{x}_{1}$-axis along the in-plane [001]-direction. $K_{p}$ and $K_{s}$ are the strain-induced uniaxial in-plane and out-of-plane anisotropy constants. The signs in Eq. (2) are chosen following the usual convention that a positive sign denotes an easy axis for the corresponding anisotropy constant. The symmetry axis for the in-plane anisotropy is the [001]-axis. By performing the appropriate tensor rotations for $\left(b_{i j}\right)$ and identifying the appropriate terms, we find for the (110)-orientation:

$$
\begin{gathered}
K_{p}=\left(b_{11}-b_{12}\right)\left(-\epsilon_{1}+\epsilon_{2} / 2+\epsilon_{3} / 2\right)+b_{44}\left(\epsilon_{2}-\epsilon_{3}\right) \\
K_{s}=2 b_{44}\left(\epsilon_{2}-\epsilon_{3}\right)
\end{gathered}
$$

In order to quantitatively compare the calculations to experimental data we need to obtain the magnetoelastic coefficients $b_{11}-b_{12}$ and $b_{44}$. Because these or derived constants for pure fcc Co do not exist in the literature, we have used values obtained for Co-rich, fcc Pd-Co alloys [17]. The magnitudes of the tabulated magnetostriction constants, $\lambda_{100}=-2 / 3\left(b_{11}-b_{12}\right) /\left(c_{11}-c_{12}\right)=130 x$ $10^{-6}$ and $\lambda_{111}=-1 / 3 \mathrm{~b}_{44} / \mathrm{c}_{44}=-65 \times 10^{-0}$, are found to be independent of the Co concentration above $60 \%$ and hence should provide a reasonable estimate for pure fcc Co.

Fig. 2 shows the obtained values for $\mathrm{K}_{\mathrm{p}}$ as a function of the relative Co layer thickness for the $\mathrm{Co} / \mathrm{Pt}$ and the $\mathrm{Co} / \mathrm{Pd}$ system. The positive sign of $\mathrm{K}_{\mathrm{s}}$ denotes that the surface normal is an easy axis for the out-of-plane anisotropy, and the positive sign of $K_{p}$ denotes that the [001]axis is an easy axis for this anisotropy. The finding of an easy [001]-axis for $C_{0}$ is unique to ultrathin, coherently strained $\mathrm{Co}(110)$ layers and caused by the anisotropic magnetoelastic interaction.

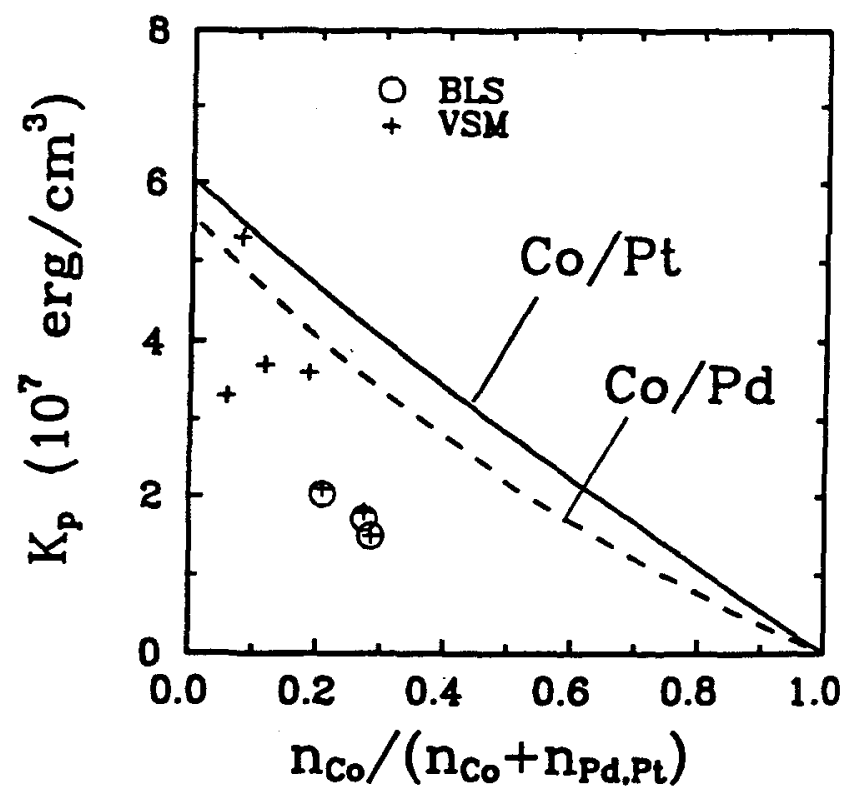

Fig. 2: Calculated in-plane $\left(\mathrm{K}_{\mathrm{p}}\right)$ anisotropy constants as a function of the relative Co layer thickness $\mathrm{n}_{\mathrm{Cg}} /\left(\mathrm{n}_{\mathrm{Cp}}+\right.$ $\mathbf{n}_{\mathbf{P d}, \mathrm{Pf}_{f}}$ ) for $\mathrm{Co} / \mathrm{Pt}$ superlattices (full lines) and Co $\mathrm{Pd}$ superlattices (dashed lines). ${ }^{\mathbf{n}} \mathrm{Ca}, \mathbf{P d}, \mathbf{P t}$ is the number of atomic layers per Co,Pd and Pt layer. Experimental data for Co/Pt is taken from Refs. [19,20] as listed in Table 1.

Experimental data obtained by VSM is available for a few $\mathrm{Co} / \mathrm{Pt}$ and $\mathrm{Co} / \mathrm{Pd}$ samples. Lin et al. [18] find for a $\mathrm{Co} / \mathrm{Pt}$ superlattice with $\mathrm{d}_{\mathrm{Co}}=3.7 \AA$ and $\mathrm{d}_{\mathrm{Pt}}=18.0 \AA$ an anisotropy constant of $K_{p}=3.6 \times 10^{7} \mathrm{erg} / \mathrm{cm}^{3}$ which compares rather well to our calculated value of $4.9 \times 10^{7}$ $\mathrm{erg} / \mathrm{cm}^{3}$. Farrow et al. measured for a number of (110)oriented $\mathrm{Co} / \mathrm{Pt}$ superlattices the change in free energy rotating the direction of magnetization from the layer normal into the easy and hard in-plane directions, respectively $[19,20]$. An analysis of their data yields with $\mathrm{d}_{\mathrm{Pt}_{\mathrm{t}} \approx} \approx$ $25 \AA$ and $\mathrm{d}_{\mathrm{C}_{0}}$ in the range of 1.1 to $9.9 \AA$ values for $\mathrm{K}_{\mathrm{p}}$ in the range of $1.5 \times 10^{7} \mathrm{erg} / \mathrm{cm}^{3}$ to $5.3 \times 10^{7} \mathrm{erg} / \mathrm{cm}^{3}$. These results are in good agreement with our calculations, which are in the range of $4.2 \times 10^{7} \mathrm{erg} / \mathrm{cm}^{3}$ to $5.6 \times 10^{7} \mathrm{erg} / \mathrm{cm}^{3}$. The experimental and calculated data are listed in Table 1. They are shown in Fig. 2 for Co/Pt multilayers.

For $\mathrm{Co} / \mathrm{Pd}(110)$-oriented superlattices a value of $K_{p}=0.35 \times 10^{7} \mathrm{erg} / \mathrm{cm}^{3}$ has been found by Brillouin light scattering, which compares to a calculated value of 1.9 $\times 10^{7} \mathrm{erg} / \mathrm{cm}^{3}$.

Although our model predicts the correct sign and magnitude of the in-plane as well as of the out-of-plane anisotropies a more quantitative comparison is still lacking. This is partly caused by the lack of reliable magnetoelastic constants for fcc-Co. Also needed is a better understanding of the influence of interface interdiffusion and roughness on the anisotropy constants. Formations of dislocations and grain boundaries release the interface strains which in turn would decrease the related anisotropy contributions. Thus the calculated anisotropy constants presented in this analysis are regarded as upper limits for the particular system.

Any interface anisotropy contributions were neglected in the comparison. We however note that the dependence of $\mathrm{K}_{\mathrm{p}}$ on $\mathrm{d}_{\mathrm{Co}}$ would suggest a large interface anisotropy contribution in a commonly used separation of measured anisotropy values into a thickness-independent volume term and an interface term proportional to $1 / \mathrm{d}_{\mathrm{Co}}$. The available data for $K_{\mathrm{p}}$ does at present not allow for the separation between thickness-dependent magnetoelastic contributions and pure interface contributions. 
Table 1: Measured (exp.) and calculated (calc.) in-plane $\left(\mathrm{K}_{\mathrm{p}}\right)$ anisotropy constants of $(110)$-oriented $\mathrm{Co} / \mathrm{Pt}$ and $\mathrm{Co} / \mathrm{Pd}$ superlattices. The experimental data were taken from Ref. [18], as well as obtained by analyzing data on $\mathrm{K}_{\text {eff }}$ of Ref. $[19,20]$ measured by VSM, as well as measured by Brillouin light scattering.

\begin{tabular}{|l|l|l|c|c|c|}
\hline sample & $\mathrm{d}_{\mathrm{Co}}$ & $\mathrm{d}_{\mathrm{Pd}, \mathrm{Pt}}$ & $\mathrm{K}_{\mathrm{p}}(\mathrm{calc})$. & $\mathrm{K}_{\mathrm{p}}$ (exp.) & method \\
\hline & {$[\AA]$} & {$[\AA]$} & {$\left[10^{7} \mathrm{erg} / \mathrm{cm}^{3}\right]$} & {$\left[10^{7} \mathrm{erg} / \mathrm{cm}^{3}\right]$} & \\
\hline $\mathrm{Co} / \mathrm{Pt}$ & 1.1 & 20.1 & 5.6 & 3.3 & $\mathrm{VSM}$ \\
$\mathrm{Co} / \mathrm{Pt}$ & 1.6 & 21.1 & 5.5 & 5.3 & VSM \\
$\mathrm{Co} / \mathrm{Pt}$ & 2.6 & 22.0 & 5.2 & 3.7 & VSM \\
$\mathrm{Co} / \mathrm{Pt}$ & 3.7 & 18.0 & 4.9 & 3.6 & VSM \\
$\mathrm{Co} / \mathrm{Pt}$ & 6.2 & 26.0 & 4.7 & 2.1 & VSM \\
& & & & 2.0 & BLS \\
$\mathrm{Co} / \mathrm{Pt}$ & 8.2 & 24.1 & 4.3 & 1.8 & VSM \\
& & & & 1.7 & $\mathrm{BLS}$ \\
$\mathrm{Co} / \mathrm{Pt}$ & 9.9 & 27.7 & 4.2 & 1.5 & VSM \\
& & & & 1.5 & $\mathrm{BLS}$ \\
$\mathrm{Co} / \mathrm{Pd}$ & 12.3 & 10.0 & 1.9 & 0.35 & $\mathrm{BLS}$ \\
$\mathrm{Co} / \mathrm{Pd}$ & 7.9 & 10.0 & 3.0 & 0.55 & $\mathrm{BLS}$ \\
\hline
\end{tabular}

It is of central importance to check whether the concept of magnetoelastic anisotropies applies to the experimentally observed out-of-plane anisotropy contributions as well. Experimental data from Ref. [21] for epitaxial Co/Pd multilayers of different growth orientation are shown in the upper part of Fig. 3 for the lowest three crystallographic orientations in $a \mathrm{~K} \cdot \mathrm{d}_{\mathrm{Co}}$ versus $\mathrm{d}_{\mathrm{Co}}$ plot. In the lower part of Fig. 3 calculated magnetoelastic anisotropy contributions are shown. In this type of graph the intersection with the ordinate indicates an interface anisotropy contribution and the slope indicates a volume anisotropy contribution. The varying slope for the calculated values indicates that for the magnetoelastic interaction a separation into volume and interface anisotropy contributions is superficial, since the strain fields themselves depend on the layer thickness although they are typically classified as bulk properties.

From a comparison of the experimental and the calculated data displayed in Fig. 3 the following conclusions are drawn: In the experimental data a positive slope for the perpendicular anisotropy in a $\mathrm{K} \cdot \mathrm{d}_{\mathrm{Co}}$ vs. $\mathrm{d}_{\mathrm{Co}}$ plot is found for the (111)-orientation, and negative slopes for the two other orientations with the slope of the (110)orientation bisecting the slopes of the (111)- and (100)orientation. The latter feature is reproduced by the calculation. However the calculated slope for the (110)-orientation is positive in contradiction to the experimental result. We conclude that although the magnetoelastic interaction accounts for the observed large in-plane anisotropy contribution it might contribute to the out-of-plane anisotropies only a small amount which depends on the crystallographic orientation. This is also corroborated by structural investigations which show an orientation-dependent strain relaxation by dislocation formation which is largest for the (111)-oriented multilayered structures [22].

\section{INFLUENCE OF SPATIAL INHOMOGENEITIES ON MAGNETIC ANISOTROPIES}

We now focus on the problem that magnetic parameters might not be considered to be uniform throughout the layers, but that they vary locally, making the layer properties inhomogeneous. We distinguish between two
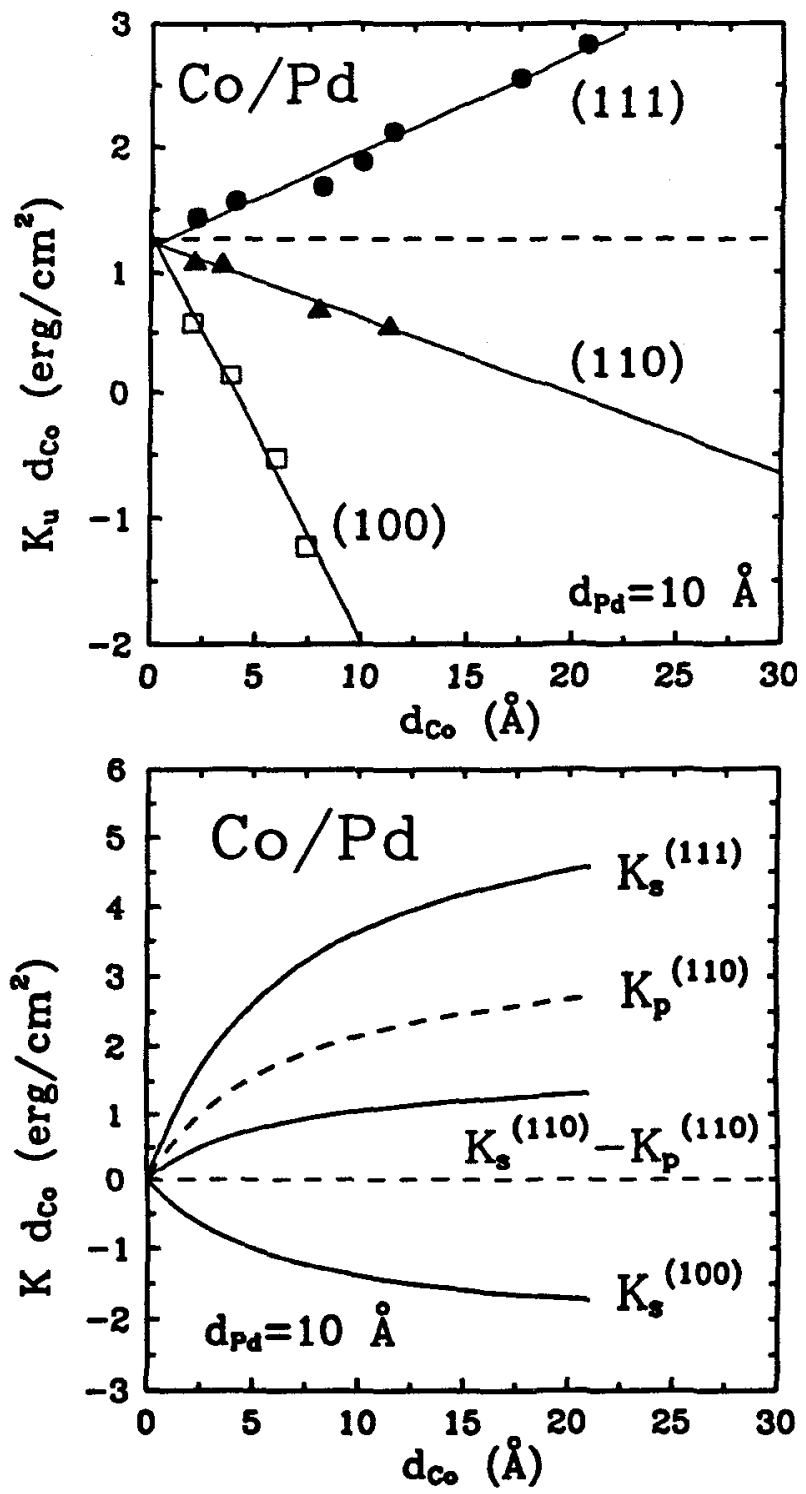

Fig. 3: Product of anisotropy constant with film layer thickness $\mathrm{d}_{\mathrm{Co}}$ as a function of $\mathrm{d}_{\mathrm{Co}}$ for (111)-, (110)- and (100)-oriented $\mathrm{Co} / \mathrm{Pd}$ multilayer structures. Upper part: Experimental data of Ref. [21]. Lower part: calculated values of the out-of-plane anisotropy (full lines) and the in-plane anisotropy (dashed line). For the (110)-orientation a contribution of the in-plane anisotropy to the free energy difference between in-plane and out-of-plane magnetization orientation must be tajen int9 account for $K_{p}>0$. Thus the values of $K_{s}^{(110)}-K_{p}^{(190)}$ are plotted for this orientation.

limiting cases depending on the length scale $\Lambda$ of lateral inhomogeneities. First, the term "inhomogeneities" refers essentially to variations of internal fields caused by magnetization-, anisotropy- or thickness- variations (corrugations), variations in the exchange constant, or variations in the interlayer thickness. For Co-based ultrathin multilayer structures variations in internal fields are mainly caused by thickness variations since the internal fields depend on the ratio of the rather large values of interface anisotropy constants to the rather small layer thickness. If $\Lambda$ is much smaller than the spin-wave wavelength $\lambda$, the spin waves depend on modified magnetic parameters which are averaged across the inhomogeneities. An example are interface anisotropies originating from smallscale interface roughness. The spin-wave wavelength 
investigated in a Brillouin light scattering experiment is of the order of $3000 \AA$. In the regime $\Lambda>\lambda$ the system is essentially homogeneous on the length scale of $\lambda$. If the sampling area, i.e. the laser spot in a Brillouin light scattering experiment, is still much larger than $\Lambda$, the observed spin wave band may also show a broadening due to sampling of a large set of spin wave spectra from different, well defined regions with different parameters and therefore with different spin wave frequencies.

In the following we present data on $\mathrm{Co} / \mathrm{Pd}$ multilayer samples prepared in a molecular beam epitaxy (MBE) system [23], and on Co/Au multilayer samples prepared using an ion-beam sputtering technique under ultrahigh vacuum conditions [24]. The Co/Au samples were postannealed for 1 hour at $200^{\circ} \mathrm{C}$ in order to atomically sharpen the interfaces due to the "backdiffusion" effect $[25,26]$. The details of the sample preparation and characterization as well as magnetometry measurements and the determination of anisotropy constants with Brillouin light scattering are described elsewhere [23,24,26-29]. The $\mathrm{Co} / \mathrm{Au}$ samples are polycrystalline and textured with the crystallographic (111)-axis normal to the layer planes. Dispersion angles are in the order of $10-15^{\circ}$. The Co/Pd samples are epitaxial and (111) oriented [23].

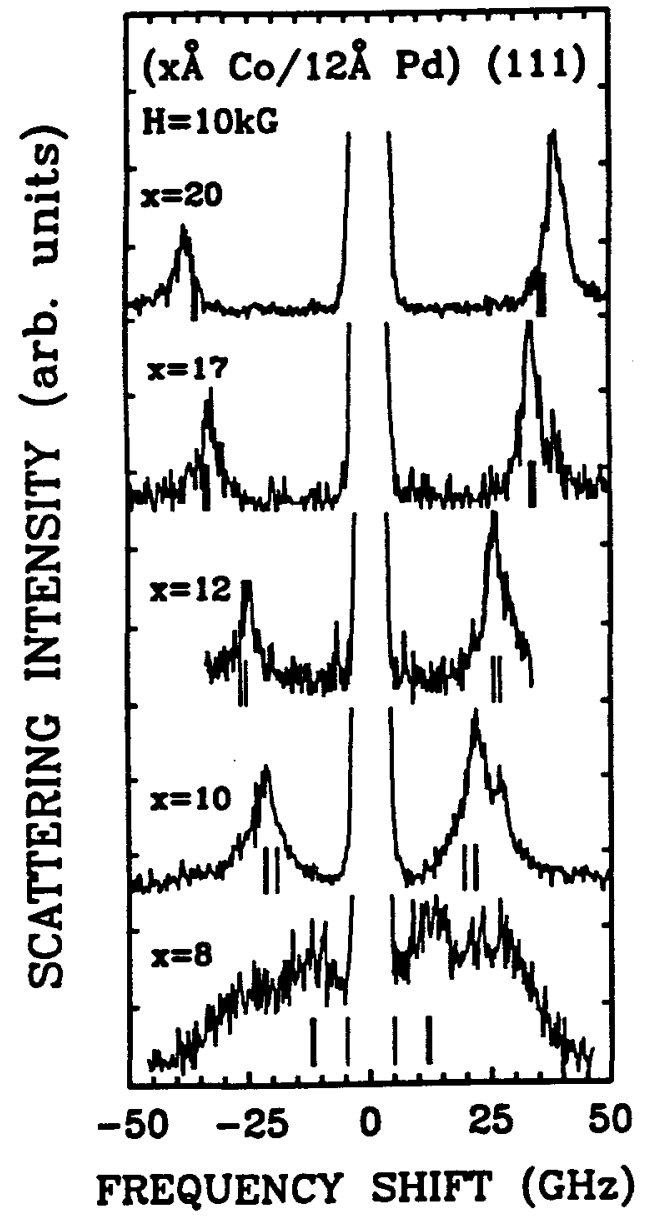

Fig. 4: Brillouin light scattering spectra of a series of MBE-prepared (111)-oriented Co/Pd superlattices with varying Co layer thickness as indicated in an external magnetic field of $10 \mathrm{kG}$.

Fig. 4 shows measured spin wave spectra of MBEgrown (111)-oriented $\mathrm{Co} / \mathrm{Pd}$ multilayers with 10-15 periods of $8-20 \AA$ Co and $12 \AA \mathrm{Pd}$, as indicated in the Figure. For comparison, the mode spectrum has been calculated [15] using the Co bulk saturation magnetization value of
$17.6 \mathrm{kG}$, the $\mathrm{g}$-factor of 2.2 , a volume anisotropy constant for hcp symmetry of $7.4 \cdot 10^{6} \mathrm{erg} / \mathrm{cm}^{3}$ and an out-of-plane surface anisotropy constant of $0.6 \mathrm{erg} / \mathrm{cm}^{2}$. The values were obtained by a fit to the center of the spin wave band [11]. For this calculation the layers are assumed to be flat with laterally constant internal fields. The obtained modes (plotted as bars in Fig. 4) are nearly degenerate in frequency and cannot be resolved on the scale of Fig. 4.

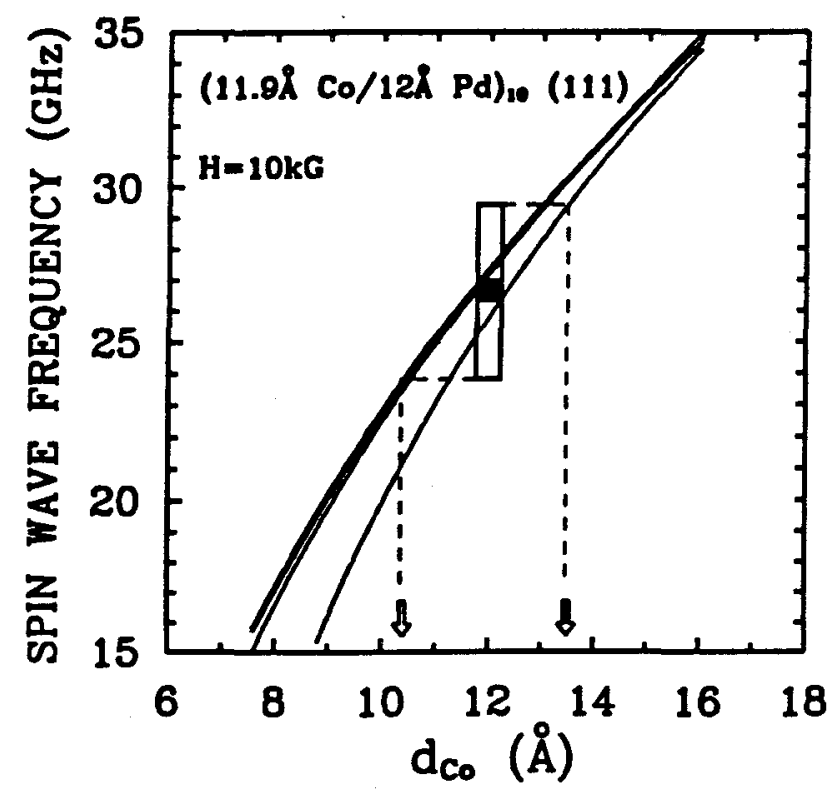

Fig. 5: Calculated spin wave frequencies of a MBE-prepared (111)-oriented Co/Pd multilayer structure (see Fig. $4, x=12$ ) with 10 bilayers of varying Co thickness and of $12 \AA$ Pd thickness in an external field of $10 \mathrm{kG}$ as a function of the Co thickness. The experimental, broad mode is shown as a bar with the intensity maximum marked with a black square. From the frequency spread of the mode (range of the bar) the corresponding change in Co layer thickness is estimated as indicated by the dashed lines.

The magnitude of the internal field variations can be estimated as follows. In Fig. 5 the frequency of the spin wave mode is calculated as a function of the Co layer thickness, $d_{\mathrm{Co}}$, for an applied magnetic field of $10 \mathrm{kG}$ using for the saturation magnetization and the uniaxial anisotropy constant the results obtained from a fit to the experimental data. By varying $\mathrm{d}_{\mathrm{Co}}$, the contribution of the interface anisotropy field to the internal field varies with $1 / \mathrm{d}_{\mathrm{Co}}$. The frequencies go to zero at $\mathrm{d}_{\mathrm{Co}} \approx 2 \AA$ (not shown in the Figure), indicating a perpendicular magnetized state for smaller values of $\mathrm{d}_{\mathrm{CO}}$. The experimentally observed line width of the peak is plotted as a bar in Fig. 5 with the center of the peak as a black square. From the length of the bar the range of spatial variations of $d_{C_{0}}$ of 10.4...13.6 $\AA$ is deduced, as illustrated in the Figure. This would translate into variations in the interface anisotropy constant of $\mathrm{k}_{\mathrm{g}}=(0.52 . .0 .68) \mathrm{erg} / \mathrm{cm}^{2}$ on assuming flat interfaces.

We will now discuss the case of multilayer structures, where the individual layer thicknesses vary from layer to layer. This might happen, e.g., due to changing deposition rates in the sample fabrication process. But even for samples with nominal identical thicknesses of all magnetic layers, the "local" thickness, i.e. the thickness on a length scale of the wavelength of the spin waves, may vary from layer to layer in the same manner as the lateral thicknesses vary as discussed above. 


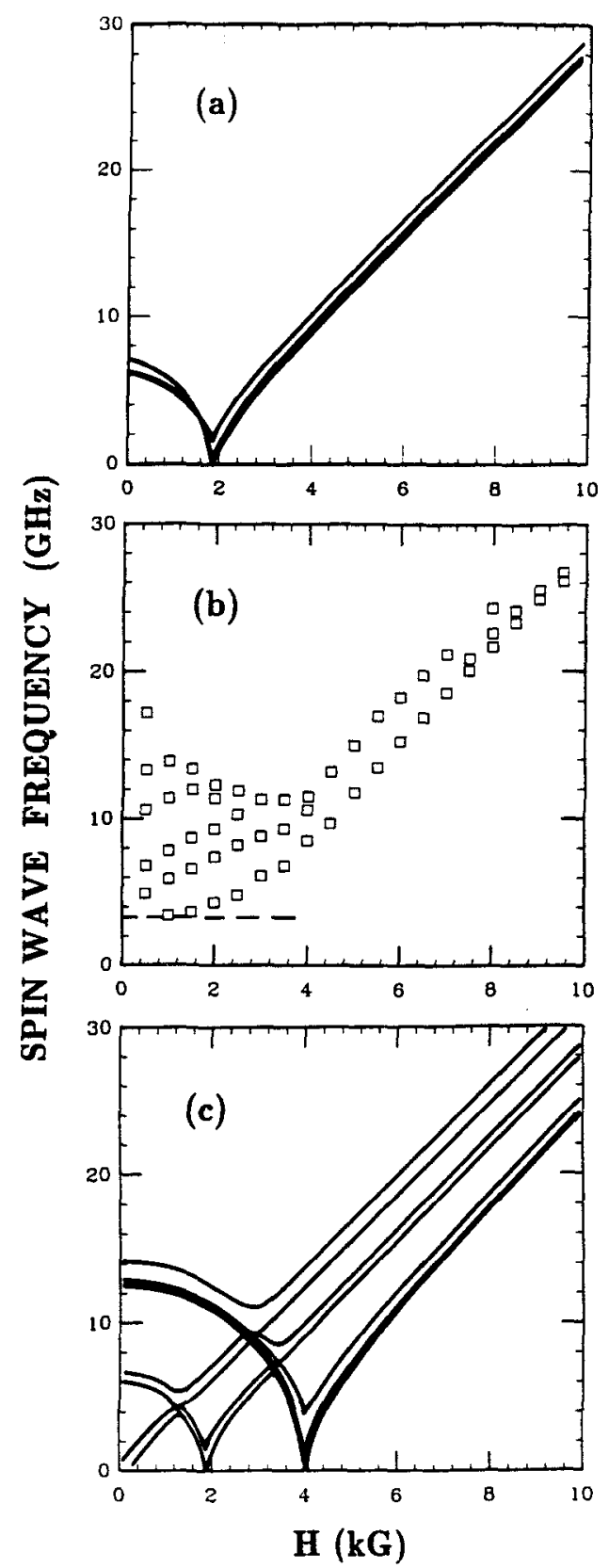

Fig. 6: Calculated (a, c) and experimental (b) spin wave frequencies as a function of the in-plane applied magnetic field. Part a) shows the calculated frequencies for a "perfect" multilayer consisting of 8 bilayers of same parameters. The thickness of magnetic and nonmagnetic layers is $10 \AA$. In b) experimental data on $\mathrm{Co} / \mathrm{Au}$ multilayers with 70 periodes and with nominal thicknesses of $\mathrm{d}_{\mathrm{Co}}=8.8$ $\AA$ and $\mathrm{d}_{\mathrm{Au}}=7.5 \AA$ is shown. The dashed line marks the threshold, below which spin wave observation is inhibited in the Brillouin light scattering experiment due to elastically scattered light. In c) the layer thicknesses of the magnetic layers are $10,9,9,11,10,9,11,9 \AA$, respectively.

We will assume a system with large interface anisotropy values, which therefore exhibits a large dependence of the spin wave frequency on the layer thickness. Without an external field the uniaxial anisotropy of each layer is assumed to be large enough to point the direction of magnetization perpendicular to the layer planes. Fig. 6a shows the calculated spin wave frequencies of a "perfect" multilayer structure of 8 bilayers with the same thickness of magnetic and nonmagnetic layers of $10 \AA$. For the magnetic layers the bulk parameters of hcp Co are assumed. With increasing in-plane applied magnetic field, the spin wave frequencies first decrease while the direction of magnetization is increasingly tilted towards the layer planes, lying in the layer planes at and above a critical field strength, $\mathrm{H}_{\text {crit }}$. For $\mathrm{H}>\mathrm{H}_{\text {crit }}$ the spin wave frequencies increase monotonically with turther increasing external field. Near $\mathrm{H}_{\text {crit }}$ the calculated spin wave frequencies show a sharp minimum with some modes going soft.

Fig. $6 \mathrm{~b}$ shows experimental data of a $(8.8 \AA \mathrm{Co} / 7.5 \AA$ $\mathrm{Au})_{\times 70}$ multilayer sample. Above $4 \mathrm{kG}$ the spin wave frequencies increase monotonically with increasing applied field indicative of the saturation magnetization lying in plane. Near $\mathrm{H}_{\text {crit }} \approx 3.5 \mathrm{kG}$ the spin wave frequencies show a broad minimum, and they are rather widely spread below $\mathrm{H}_{\text {crit. }}$. The observed behavior is in only rough qualitative agreement with the calculated field dependence of the spin wave modes shown in Fig. 6a. We will now show that by allowing the individual layer thicknesses to have a distribution about the mean thickness value the calculated spin wave properties resemble much better the experimental data.

Fig. 6c shows calculated spin wave frequencies for a multilayer consisting of 8 bilayers. The nonmagnetic layer thickness is fixed at $10 \AA$, the magnetic layer thicknesses are $10,9,9,11,10,9,11$ and $9 \AA$, respectively. For this calculation $A_{12}$ has been set to zero. For each layer, first the critical field, $\mathrm{H}_{\text {crit }}$, is calculated as well as the direction of the magnetization as a function of the applied in-plane field. $\mathrm{H}_{\text {crit }}$ varies from layer to layer due to different layer thicknesses. Then the spin wave frequencies of the multilayer stack are calculated, using the static orientation of the layer magnetizations as input data. The spin wave modes show zero frequencies at $\mathrm{H}_{\text {crit }}=0,1.9$ and $4 \mathrm{kG}$. The values of $\mathrm{H}_{\mathrm{c}}$ correspond to the chosen thickness values of the magnetic layers of 11,10 , and $9 \AA$, respectively. The obtained spin wave mode distribution resembles the experimentally observed mode spectrum (Fig. 6b) in a much better way than does the calculation assuming the same parameters for each layer, as shown in Fig. 6a. Please note that spin wave modes with frequencies smaller than about $3 \mathrm{GHz}$ (dashed line in Fig. $6 \mathrm{~b}$ ) are not accessible in the Brillouin light scattering experiment due to the overlap with elastically scattered laser light.

For a "real" multilayer structure, both thickness variations from layer to layer as well as thicknesses varying laterally due to, e.g., a mosaic spread, contribute to the effect. The $\mathrm{Co} / \mathrm{Au}$ sample, of which the spin wave data are shown in Fig. $6 \mathrm{~b}$, was prepared by postannealing the sample in order to gain atomically sharp interfaces for maximizing interface anisotropies. On the other hand, evidence has been found that the postannealing process introduces interface corrugations, which might be responsible for local, layer-to-layer thickness variations [25]. The pronounced difference in the spin wave properties between a "perfect" structure (Fig. 6a) and a "realistic" structure as described above (Fig. 6c) is already obtained for a corrugation of $\pm 1 \AA$ of each layer.

\section{CONCLUSIONS}

We have shown two major aspects of anisotropies in multilayered structures: the identification of the uniaxial in-plane anisotropy in (110)-oriented $\mathrm{Co} / \mathrm{Pd}$ and $\mathrm{Co} / \mathrm{Pt}$ multilayers as being of magnetoelastic origin, as well as spatially varying anisotropy fields due to thickness variations of the individual layers.

We would like to comment on the influence of interdiffusion and roughness on the strain fields and therefore on the anisotropy values. For very small Co layer 
thicknesses comparable to the interdiffusion length the strains are more or less equally distributed between both materials according to the Co layer thickness. If the layers are thicker, our model approach applies if modified strain fields are considered. The model is thus valid in a thickness regime larger than the interdiffusion thickness and smaller than the critical thickness needed for dislocation formation.

The length scale for determining spatial variations by Brillouin light scattering can be estimated as follows: The smallest length scale is given by the static spin correlation length, $\xi_{\text {spin }}$, which is of the order of a few tens of Angstroms for Co and Fe. Below $\xi_{\text {spin }}$ any local inhomogeneities, caused by, e.g., defects or interface roughness on the atomic scale, are averaged out in the spin system. The next characteristic length scale is given by the spinwave wavelength, $\lambda$, which here is of the order of $3000 \AA$. If the length scale of lateral inhomogeneities, $\Lambda$, is much smaller than $\lambda$, the spin waves average across the inhomogeneities and sense modified magnetic parameters. An example is the modification of interface anisotropies due to roughness, which is essentially obtained by averaging the demagnetizing fields across the interface corrugation profile. If the spin wave wavelength is of the order of the length scale $\Lambda$ of lateral inhomogeneities, a large modification of the spin-wave mode spectrum takes place due to propagation of spin waves from one local environment to the next with different magnetic parameters. For $\Lambda>\lambda$ the sample appears locally homogeneous and the laser spot in the Brillouin light scattering experiment, which is still larger in diameter than $\Lambda$, averages over regions each supporting a spin wave spectrum of a homogeneous material.

The characteristic length scale perpendicular to the layers is directly given by the layer thicknesses. Across each magnetic layer the fluctuating components of the magnetization and the magnetic field are essentially constant for dominantly dipolar modes in the layer thickness regime of a few Angstroms. The spin wave modes sense those internal fields, which depend largely on the interface anisotropy constants and the layer thickness.

\section{ACKNOWLEDGEMENTS}

We would like to thank B.N. Engel, C.M. Falco, R.F.C. Farrow and D. Weller for sample supply and discussions and R.L Stamps for discussions and the calculations for Fig. 6. Support by the Deutsche Forschungsgemeinschaft through SFB 341, NATO and NSERC of Canada is gratefully acknowledged.

\section{REFERENCES}

[1] H.J.G. Draaisma, W.J.M. de Jonge, J. Magn. Magn. Mater. 66, 351 (1987).

[2] H.J.G. Draaisma, F.J.A. den Broeder, W.J.M. de Jonge, J. Appl. Phys. 63, 3479 (1988).

[3] F.J.A. den Broeder, D. Kuiper, H.C. Donkersloot, W. Hoving, Appl. Phys. A 49, 507 (1989).

[4] B.N. Engel, C.D. England, R.A. Van Leeuwen, M.H. Wiedmann, C.M. Falco, Phys. Rev. Lett. 67, 1910 (1991).

[5] C.H. Lee, R.F.C. Farrow, C.J. Lin, E.E. Marinero, C.J. Chien, Phys. Rev. B 42, 11384 (1990).

[6] W.B. Zeper, H.W. van Kesteren, B.A.J. Jacobs, J.H.M. Spruit, P.F. Carcia, J. Appl. Phys. 70, 2264 (1991).

[7] F.J.A.M. Greidanus, W.B. Zeper, F.J.A. den Broeder, W.F. Godlieb, P.F. Carcia, Appl. Phys. Lett. 54, 2481 (1989).
[8] W.B. Zeper, F.J.A.M. Greidanus, P.F. Carcia, C.R. Fincher, J. Appl. Phys. 65, 4971 (1989).

[9] S. Hashimoto, Y. Ochiai, K. Aso, J. Appl. Phys. 67, 2136 (1990).

[10] B. Hillebrands, J.R. Dutcher, Phys. Rev. B, in press.

11] B. Hillebrands, J.V. Harzer, R.L. Stamps, G. Günthe rodt, D.Weller, B.N. Engel, C.M. Falco, to be published.

[12] B. Hillebrands, A. Boufelfel, C.M. Falco, P. Baumgart, G. Güntherodt, E. Zirngiebl, J.D. Thompson, J. Appl. Phys. 63, 3880 (1988).

[13] B. Hillebrands, G. Güntherodt, in: Ultrathin Magnetic Structures Vol II, J.A.C. Bland, B. Heinrich (eds.), Springer Verlag (Heidelberg, Berlin, London, New York, Tokyo, 1993).

[14] P. Grünberg, Light Scattering in Solids V, M. Cardona, G. Güntherodt (eds.), Springer Ser. Topics Appl. Phys. 66, 303 (1989).

[15] B. Hillebrands, Phys. Rev. B 41, 530 (1990).

16] R. Mock, B.Hillebrands, and J.R. Sandercock, J. Phys. E 20, 656 (1987).

[17] H. Fujiwara, H. Kadomatsu, T. Tokunaga, J. Magn. Magn. Mater. 31-34, 809 (1983).

[18] C.-J. Lin, G.L. Gorman, C.H. Lee, R.F.C. Farrow, E.E. Marinero, H.V. Do, H. Notarys, C.J. Chien, J. Magn. Magn. Mater. 93, 194 (1991).

[19] D. Weller, R.F.C. Farrow, C.H. Lee, R.F. Marks, G.R. Harp, to be published.

[20] R.F.C. Farrow, C.H. Lee, R.F. Marks, G. Harp, M. Toney, T.A. Rabedeau, D. Weller, H. Brāndle, Proceedings of the Nato Advanced Research Workshop on Magnetism and Structure in Systems of Reduced Dimension, Institut d'Etudes Scientifiques de Cargèse, France, Plenum Press (1993), in press.

[21] B.N. Engel, C.D. England, R.A. Van Leeuwen, M.H. Wiedmann, C.M. Falco, J. Appl. Phys. 70, 5873 (1991).

[22] C.J. Chien, R.F.C. Farrow, C.H. Lee, C.J. Lin, E.E. Marinero, J. Magn. Magn. Mater. 93, 47 (1991).

[23] C.D. England, B.N. Engel, C.M. Falco, J. Appl. Phys. 69, 5310 (1991).

[24] K. Spörl, D.Weller, G. Rupp, J. Magn. Magn. Mater. 83, 91 (1990).

[25] F.J.A. den Broeder, D. Kuiper, A.P. van de Mosselaer, and W. Hoving, Phys. Rev. Lett. 60, 2769 (1988).

[26] K. Spörl, D. Weller to be published.

27] J.V. Harzer, B. Hillebrands, R.L. Stamps, G. Günthe rodt, D. Weller, Ch. Lee, R.F.C. Farrow, E.E. Marinero, J. Magn. Magn. Mater. 104-107, 1863 (1992).

[28] B.N. Engel, C.D. England, R. van Leeuwen, M. Nakada, C.M. Falco, J. Appl. Phys. 69, 5643 (1991).

[29] B. Hillebrands, P. Krams, K. Spörl, D. Weller, J. Appl. Phys. 69, 938 (1991). 